\title{
The development of bioresorbable composite polymeric implants with high mechanical strength
}

\section{Citation}

Sharma, Upma, Danny Concagh, Lee Core, Yina Kuang, Changcheng You, Quynh Pham, Greg Zugates, et al. 2017. "The Development of Bioresorbable Composite Polymeric Implants with High Mechanical Strength." Nature Materials 17 (1) (November 20): 96-103. doi:10.1038/ nmat5016.

\section{Published Version}

doi:10.1038/nmat5016

\section{Permanent link}

http://nrs.harvard.edu/urn-3:HUL.InstRepos:34707485

\section{Terms of Use}

This article was downloaded from Harvard University's DASH repository, and is made available under the terms and conditions applicable to Other Posted Material, as set forth at http:// nrs.harvard.edu/urn-3:HUL.InstRepos:dash.current.terms-of-use\#LAA

\section{Share Your Story}

The Harvard community has made this article openly available.

Please share how this access benefits you. Submit a story.

\section{Accessibility}


5 The development of bioresorbable composite polymeric implants with high mechanical strength

$8 \quad$ Upma Sharma1 PhD, Danny Concagh ${ }^{1}$ MS, Lee Core ${ }^{1}$ MSE, Yina Kuang ${ }^{1}$ PhD, Changcheng 9 You ${ }^{1}$ PhD, Quynh Pham ${ }^{1}$ PhD, Greg Zugates ${ }^{1}$ PhD, Rany Busold ${ }^{1}$ BS, Stephanie Webber ${ }^{1}$ BS, 10 Jonathan Merlo ${ }^{1} \mathrm{BS}$, Robert Langer $\mathrm{PhD}^{2}$, George Whitesides $\mathrm{PhD}^{3}$, Maria Palasis ${ }^{1} \mathrm{PhD}$

${ }^{1} 480$ Biomedical, Inc., Watertown, MA 02472, USA

23 Address for reprints and manuscript correspondence:

24 Maria Palasis, PhD

25480 Biomedical, Inc.

26480 Arsenal Street

27 Watertown, MA 02472

28 mpalasis@480biomedical.com 
32 Implants for the treatment of tissue defects should mimic the mechanical properties of the native 33 tissue of interest and should be resorbable as well as biocompatible. In this work, we developed 34 a scaffold from variants of poly-L-glycolic acid which were braided and coated with an elastomer 35 of poly (glycolide-co-caprolactone) and crosslinked. The coating of the scaffold with the elastomer led to higher mechanical strength in terms of compression, expansion and elasticity compared to 37 braids without the elastomer coating. These composite scaffolds were found to have expansion 38 properties similar to metallic stents, utilizing materials which are typically much weaker than metal. 39 We optimized the mechanical properties of the implant by tuning the elastomer branching 40 structure, crosslink density, and molecular weight. The scaffolds were shown to be highly 41 resorbable following implantation in a porcine femoral artery. Biocompatibility was studied in vivo 42 in an ovine model by implanting the scaffolds into femoral arteries. The scaffolds were able to 43 support an expanded open lumen over 12 months in vivo and also fully resorbed by 18 months in 44 the ovine model. 
Many soft tissues in the body undergo significant motion or experience substantial pressure. Strong, elastic, bioresorbable implants could be useful in cartilage repair, vascular grafts, sinusitis treatment, and treatment of pediatric conditions. A major limitation of medical implants used to treat these tissues is the lack of materials that mimic the strength and elasticity of the native tissue. Beyond the mechanical properties, an ideal medical implant would also be resorbable, to provide utility only until the native tissue has healed.

To illustrate the capability of our scaffold, we focused on arterial disease where strong, bioresorbable materials have been touted as the wave of the future [1-4]. Here, bioresorbable scaffolds provide temporary strength - holding a vessel at an expanded diameter and resisting vessel recoil only until healing has occurred - while eliminating a permanent foreign body. Balloon expandable, polymeric, bioresorbable scaffolds are fabricated from monolithic, highly-crystalline, oriented, extruded tubes of polymers to achieve the necessary mechanical properties $[1,4,5]$. While such scaffolds have found applications in coronary arteries, these devices would fail in patients with peripheral arterial disease, whereby the vessels (such as the superficial femoral artery) undergo significant motion resulting in kinking and fracturing of the stiff devices. As a result, vessels with significant motion are currently treated using self-expanding metal devices, which have the required strength to resist vessel recoil and are designed with elasticity and flexibility to withstand the forces resulting from vessel motion. Despite this, self-expanding metal devices have high fracture rates associated with stent restenosis that significantly lowers vessel patency [6].

An ideal stent in vessels experiencing significant motion would couple the benefits of selfexpanding metal devices (strength and elasticity of native tissue) with those of bioresorbable devices (no permanent foreign body). Currently, no such device exists. The key challenge in creating any self-expanding implant is the need for the implant to be strong, elastic, and biocompatible. Designing a self-expanding device that is also bioresorbable is particularly challenging due to the limited strength of available materials. For example, "strong" bioresorbable polymers have tensile properties that are only one-tenth those of the metals used in existing selfexpanding devices. This design challenge is further complicated by the environment of the vasculature, where materials have elicited higher inflammatory responses relative to other body locations [7-11]. A final design complexity is that commonly used bioresorbable polymers are prone to stress relaxation. That is, when the device is crimped into a catheter for delivery to the target vessel, the strain on the device can lead to permanent deformation.

We hypothesized that we could create a self-expanding, bioresorbable implant utilizing a unique composite design. This design would consist of fiber braid (strong, highly oriented fibers in a design that enables bending) coated with an elastomer. The thermoset elastomer would be cured on the braid at the fully-expanded diameter to provide a mechanism for the implant to "spring back" or self-expand to its fabricated diameter by constraining the points of intersection of the braid. Utilizing this composite design, we have created an implant with radial force properties equivalent to metal, using "weak" polymeric materials that fully resorb.

\section{Benchmark self-expanding designs and the limitations of polymeric materials}

Our initial design efforts for a bioresorbable polymer implant focused on braiding fibers 
orientation, in order to maximize the modulus of the materials. Despite these processing efforts, the PGA, L-PLA, and their copolymer poly(L-lactide-co-glycolide) (L-PLGA) fibers had significantly less mechanical stiffness than the stainless steel or nickel titanium (NiTi) materials that are used to manufacture two benchmark self-expanding metal stent devices (Figure 1A) [12]. The Wallstent ${ }^{\circledR}$ (Boston Scientific Corporation) utilizes stainless steel wires in a braided design, imparting flexibility and elasticity on this stiff, inelastic material (e.g. 316L stainless steel has a tensile modulus $=200 \mathrm{GPa}$, elongation-to-break $<1 \%$ ) while S.M.A.R.T® (Cordis Corporation) stent is fabricated from super-elastic metals, such as nickel titanium (NiTi), that possesses both stiff and elastic material properties (e.g. NiTi used in S.M.A.R.T® stent has tensile modulus $=40$ $75 \mathrm{GPa}$, elongation-to-break $=10-15 \%)$. These differing approaches have resulted in stent designs with a range of mechanical properties. The radial stiffness (RRF), a measure of the stent's ability to withstand compression from a vessel, for the S.M.A.R.T. $\AA$ and Wallstent $\circledast$ stents are 411 and $140 \mathrm{mmHg}$, respectively; the chronic outward force (COF), a measure of a stent's ability to expand the vessel wall, for the S.M.A.R.T. ${ }^{\circledR}$ and Wallstent ${ }^{\circledR}$ stents are $208 \mathrm{mmHg}$ and 68 $\mathrm{mmHg}$, respectively (Figure 1B). These metal devices also recover fully to their initial diameter after deployment, as there is no stress relaxation leading to plastic deformation.

Base braid designs fabricated from optimally processed PLGA fibers had insufficient mechanical properties when compared to the above benchmark devices, consistent with the observations of others (Figure 1B) [13]. In addition to the weak mechanical properties, stress relaxation was also observed when performing simulated deployments of the base braid. That is, the process of crimping the base braid to a 7French catheter to enable delivery to the target vessel and then expanding the device 10 minutes later led to significant stress relaxation of the polymers and permanent deformation of the device - resulting in a $25 \%$ reduction in the diameter of the braid, consistent with the results of others [14, 15]. We hypothesized that a successful bioresorbable, self-expanding implant should have mechanical properties similar to these benchmark metal devices.

\section{Design and optimization of the elastomer}

To impart desired mechanical properties to the base braid, we developed a bioresorbable elastomer material (that would be used to coat the base braid). We hypothesized that the combination of the fibers comprising the base braid, coupled with an overlying elastomer coating that constrains the intersection points of the braid (Figure 2A-C) would result in an implant with the ability to self-expand into a flexible, elastic structure with high radial stiffness (Figure 2D, E). Sufficient elasticity of the coating would be required to withstand the range of diameters experienced during the crimping and deployment process (e.g., manufactured diameter $\sim 7 \mathrm{~mm} \rightarrow$ diameter in catheter $\sim 1.9 \mathrm{~mm} \rightarrow$ expanded diameter in vessel $\sim 6 \mathrm{~mm}$ ).

We developed the elastomer leveraging well-characterized, biocompatible materials from the PLA, PGA, and polycaprolactone (PCL) families. These materials degrade via hydrolysis into metabolites that can be safely eliminated from the body [16]. Because of this safe route of elimination, these materials have been widely used in the body as sutures, orthopedic tissue fixation devices, and drug delivery systems [17]. Additionally, this class of polymers provides the ability to modulate the mechanical properties and absorption profile by combining the monomers at various compositions [18]. 
The glass transition temperature $\left(T_{g}\right)$ of an elastomer is critical in determining its elasticity. To ensure that the elastomer is not in the glassy state during crimping or deployment, its $T_{g}$ must be less than room and body temperature. This requirement necessitated incorporation of $\varepsilon^{-}$ caprolactone into the elastomer, due to the low $\mathrm{T}_{g}$ of its polymers $\left(-60^{\circ} \mathrm{C}\right)$; in contrast, the $\mathrm{T}_{\mathrm{g}}$ of lactide and glycolide polymers is above body temperature. However, homopolymers of $\varepsilon^{-}$ caprolactone are highly crystalline and prone to permanent deformation. Therefore, in addition to $\varepsilon$-caprolactone, we also selected glycolide and lactide as the building blocks of our initial elastomer to provide a fast resorbing, highly elastic material.

Initial elastomers were synthesized as linear pre-polymers of poly(glycolide-cocaprolactone) (PGCL). We prepared films from this prepolymer and characterized their mechanical properties. This elastomer demonstrated a high elongation-to-break, although it was prone to high plastic deformation. As a result, when the stretched elastomer was released it would not recover to its original dimensions. We hypothesized this deformation was due to irreversible alignment of polymer chains under tensile forces. To decrease this deformation, we incorporated a four-arm branched initiator, pentaerythritol, into the reaction to create a four-arm branched prepolymer of PGCL (Figure 2F). The four-arm structure provided "crosslink" points in the prepolymer, helping to overcome plastic deformation and providing mechanical strength to the elastomer. Elasticity and deformation of the elastomer were further optimized by controlling the crosslink density of the elastomer. A tightly crosslinked elastomer, while strong, yielded a low elongation-to-break. In contrast, a loosely crosslinked system behaved similarly to the noncrosslinked linear polymer; it had high elongation-to-break but was prone to plastic deformation. Figure 3A illustrates the mechanical properties of two, four arm PGCL cross linked elastomers prepared: one with low elongation-to-break and one with high elongation-to-break. The molecular weights of the prepolymers for these elastomers are 20,000 and $40,000 \mathrm{~g} / \mathrm{mol}$, respectively, and both elastomers were fabricated at a prepolymer-to-hexamethylene diisocyanate (HDI) ratio of 8:1 (wt/wt). Figure 3B and C show the morphology of base braids coated with these elastomers after they have been crimped and deployed from a 7 French catheter. The low elongation-to-break elastomer coated implants results in visible cracking of the material while the high elongation-tobreak elastomer coated implant had no cracking.

Crosslink density was controlled by adjusting both molecular weight of the prepolymer and the amount of crosslinker used to prepare the elastomer. We created a series of elastomers using PGCL of different molecular weights (e.g., 20,000 and 100,000 $\mathrm{g} / \mathrm{mol}$ ) with various amounts of HDI crosslinker (e.g., ratio of elastomer to HDI of 20:1 to 20:4 by weight). Mechanical measurements demonstrated that higher molecular weight prepolymers with optimized cross-link density yielded elastomers with high elongation-to-break of $300 \%$ or greater (Supplemental Table 1).

Elastomers were further characterized to understand the contribution of plastic deformation to the material elongation. We hypothesized that an elastomer with high plastic deformation would lead to irreversible stretching during crimping, resulting in incomplete recovery of the elastomer-coated implant. We measured plastic deformation using cyclic mechanical loading. In this process, tensile testing was performed on dogbone-shaped samples for five consecutive cycles up to $300 \%$ strain, and the permanent deformation was defined as the residual 
strain at the beginning of the fifth cycle. Crosslink density and molecular weight were optimized to ensure less than $25 \%$ permanent deformation.

In summary, we created fully resorbable elastomers, leveraging well-characterized, biocompatible building blocks. We were able to "lock" the intersection points of fibers on a base braid, by coating the braid with a thermoset elastomer. The coating is intended to restrain but not restrict pivoting of the fiber struts (if the coating is too restrictive, then the fiber struts would bend, potentially leading to buckling, deformation, or poor recovery). To optimize mechanical properties, we incorporated polymer branching along with modulation of cross-link density and molecular weight. We selected an optimized, branched PGCL elastomer with greater than $300 \%$ elongationto-break and less than $25 \%$ permanent deformation for further study. Doing so yielded a strong implant from "weak" materials.

Our composite structure created an implant with unique properties relative to current polymeric resorbable stents that utilize monolithic, highly-crystalline, oriented, extruded polymer tubes. These devices include Remedy (Kyoto Medical), Bioresorbable Vascular System (Abbott), and Desolve (Elixir) stents. Although the latter two devices have good radial strength, they have minimal COF and therefore are not self-expanding. This data highlights the uniqueness of the design created herein.

\section{Acute mechanical properties}

The optimized PGCL elastomer was applied to the base braid and cured, creating a polyester with urethane crosslinks (polyester/polyurethane) as shown in Figure $2 \mathrm{~F}$. The resulting implant has a strut diameter and angle of approximately 125-175 micron and 120-130 respectively, with a cell size between $0.024-0.030 \mathrm{~mm}^{2}$. An implant comprised of 10:90 L-PLGA fibers coated with PGCL (10:90/PGCL) elastomer resulted in a device with strong mechanical properties, exhibiting an RRF $=712 \pm 45 \mathrm{mmHg}$ and $\mathrm{COF}=151 \pm 5 \mathrm{mmHg}$. In contrast, the same braid without an elastomer coating had mechanical properties of RRF $=90 \pm 5 \mathrm{mmHg}$ and COF $=28 \pm 3 \mathrm{mmHg}$ (Figure 1B). These results demonstrate that the elastomer coating substantially improved the mechanical properties of the base braid, achieving mechanical properties similar to benchmark self-expanding metallic stents but utilizing fully resorbable materials (Figure 1B). Radial force testing under multiple cycles indicated that at a $4.5 \mathrm{~mm}$ diameter, the maximum acute plastic deformation response decreased the RRF by $\sim 25 \%$ while the COF remains essentially unchanged. Over a range of target vessel diameters, the RRF is relatively constant with values between $890 \pm 54$ to $823 \pm 61 \mathrm{mmHg}$ while the COF ranges from $243 \pm 9$ to $118 \pm 7 \mathrm{mmHg}$ (Supplemental Figure 1). These results are particularly remarkable when considering that the mechanical properties of the starting materials for the bioresorbable were considerably weaker than those used in metal stents. Application of the optimized elastomeric coating had an additional benefit - an acute recovery greater than $95 \%$ of the manufactured diameter was demonstrated, overcoming the acute stress relaxation of the base braid during crimping and deployment. To avoid the long-term effects of prolonged duration in a crimped state on the ability of an implant to self-expand, our implants will be packaged in an uncrimped state along with a delivery system that contains a mechanism to crimp the implant into a catheter immediately prior to its use.

\section{Chronic properties and vascular biocompatibility}


Beyond the acute phase, a critical characteristic of any self-expanding, resorbable implant is the biocompatibility throughout its resorption. The materials resorb via a hydrolytic resorption mechanism through random scission of the ester linkage in the polymer's backbone as shown in Supplemental Figure 2. The major resorption products from this hydrolytic process are the small molecules lactic acid, glycolic acid, and 6-hydroxyhexanoic acid from the PLA, PGA and PCL polymer segments, respectively $[19,20]$. These small molecules or further degradants of these small molecules then enter the tricarboxylic acid cycle and are eventually eliminated from the body as carbon dioxide and water. The urethane/urea segments make up less than $4 \%$ by weight of the implant and degrade via an oxidative mechanism giving rise to the small molecule hexamethylenediamine (HDA) [21]. HDA is then eliminated from the body via urine [22, 23].

To assess the biocompatibility of the 10:90/PGCL design, devices fabricated with $6 \mathrm{~mm}$ diameter were implanted in ilio-femoral arteries in swine. Placement was successful for all devices in the target 4-5 $\mathrm{mm}$ vessel diameter as measured by angiography, confirming the acute mechanical performance of the device in vivo. Animals were sacrificed at 30 or 90 days, based on the expected resorption time of the 10:90/PGCL device of 3 - 4 months. At both follow up time points, no evidence of implant migration, thrombosis, dissection, or aneurysm was observed. All vessels were open and patent by angiography (defined as less than 50\% binary stenosis), although some level of stenosis at 30 days was noted (Figure 4A-D). While angiographic results were promising, histologic analysis demonstrated a significant inflammatory response and the presence of granuloma at 30 days (Figure 4E, F, Supplemental Table 2). Disruptions of the internal elastic lamina (IEL) and external elastic lamina (EEL) were frequent and associated with inflammation, indicating substantial vessel injury. A significant neoinitimal response was also observed at 30 days, with some resolution by 90 days. Moderate resorption was observed by histology at 30 days, and substantial resorption was seen at 90 days. The inflammatory response and the associated granuloma were attributed to the fast resorption of the implant. Overall, the biocompatibility of this implant was deemed unacceptable.

To study the impact of slowing the resorption of the device on vascular compatibility, two additional designs were evaluated: a 75:25 L-PLGA base braid coated with the same PGCL elastomer (75:25/PGCL) and a 75:25 L-PLGA base braid coated with a PLCL elastomer (75:25/PLCL). Doing so enabled us to study the impact of slowing the resorption of the base braid only (i.e., comparison of $10: 90 / P G C L$ with $75: 25 / P G C L$ ) versus slowing the resorption of the elastomer (i.e., comparison of the 75:25/PGCL with 75:25/PLCL). Both of these strategies utilized the slower resorption of L-lactide as compared to glycolide [12]. Resorption time of these base braids and implants were compared using an in vitro accelerated resorption assay. In this assay, one day of accelerated resorption corresponded to approximately one-week of real time resorption. Comparison of the resorption time of the two base braids (75:25 and 10:90) and the three coated devices (10:90/PGCL, 75:25/PGCL, 75:25/PLCL) demonstrated that the 10:90 devices resorbed more quickly than the 75:25 devices (Figure 5), as expected based on the higher glycolide concentration [12]. The elastomer coating on the 10:90/PGCL implant slowed its resorption, relative to the 10:90 base braid. Similarly, the PLCL coating on the 75:25/PLCL device decreased the resorption relative to the $75: 25$ base braid. In contrast, the 75:25/PGCL device had similar resorption to the uncoated base braid. These results indicate that the resorption time of the 75:25 and the PGCL elastomer were likely similar, leading to no change in overall resorption 
255

256

257

258

259

260

261

262

263

264

265

266

267

268

269

270

271

272

273

274

275

276

277

278

279

280

281

282

283

284

285

286

287

288

289

290

291

292

293

294

295

time with the coating. Based on these results, and the correlation between accelerated and real time resorption, the 10:90/PGCL should resorb in vitro in approximately 4 months - consistent with our in vivo results. Extrapolating from in vitro results, the anticipated resorption times for the 75:25/PGCL and the 75:25/PLCL were $6-7$ months and $9-10$ months, respectively.

When implanted in swine, all vessels with the $75: 25 / P G C L$ and $75: 25 / P L C L$ devices remained patent by angiography at 30,90 , and 180 days. Additionally, all vessels were fully endothelialized by 30 days. In the 75:25/PGCL group, significant in vivo resorption had occurred at 90 days (consistent with in vitro predictions), as evidenced histologically by decreasing size of implant struts and penetration of cells into the device remnants over time. This resorption coincided with a strong inflammatory response with the presence of granuloma. By 180 days, this inflammatory response had partially subsided, but we deemed the device incompatible based on the 90 day response. Examination of the slowest degrading 75:25/PLCL device revealed excellent histological compatibility (Figure 6A). Only a minimal-mild inflammation was observed at 30 and 90 days; disruptions of the internal elastic lamina (IEL) and the external elastic lamina (EEL) were rare. The overall stenosis observed in this device was mild and considered to be acceptable (average diameter stenosis measured by angiography of $9.1 \pm 2.7 \%$ and $7.4 \pm 5.6 \%$ at 90 and 180 days, respectively). Minimal resorption was observed at 90 days, though occasional cell infiltration was observed histologically in the implant struts indicating resorption had initiated. Significant resorption occurred at 180 days, with only small remnant particles of the device remaining. In contrast to the PGCL devices, this resorption was accompanied by a modest amount of inflammation and minimal IEL/EEL disruption. These results suggest excellent compatibility of the 75:25/PLCL bioresorbable implant throughout the resorption process. Detailed quantitative assessments of the implantation and semi-quantitative comparison of injury, inflammation, and fibrin between groups and timepoints are provided in Supplemental Table 2.

Overall, the three implant designs tested demonstrated distinct resorption profiles. These results indicate the importance of the resorption properties of the device, both the base braid and the elastomer, on its vascular compatibility. These results are consistent with others who have demonstrated the importance of controlling the rate of implant resorption within the initial critical vessel healing period [24].

\section{Addressing elastic recoil}

It has been previously hypothesized that a bioabsorbable stent should not lose mechanical properties until the vessel has fully remodeled to avoid chronic recoil (estimated time $\sim 90$ days). While the 75:25/PLCL device demonstrated excellent compatibility in animals, bench data indicated that this device exhibited minimal COF after 28 days (Supplemental Figure 3). In an effort to extend the retention of mechanical properties, an additional variant of the implant was studied that used 85:15 L-PLGA fiber as the base braid material with a PLCL elastomer coating, $85: 15 / \mathrm{PLCL}$, and were able to demonstrate measurable properties out to 3 months (Supplemental Figure 3). Fatigue testing on this design showed mechanical integrity out to 22 weeks (Supplemental Figure 4).

We conducted an additional pre-clinical study to examine the biocompatibility of the 75:25/PLCL and 85:15/PLCL devices throughout their full resorption. In this study, we utilized the 
femoral and profunda vessels in an ovine model to assess compatibility in a second species and vessel bed. The ovine model was selected because it provides longer and larger vessels in the legs, representing a closer anatomy to human superficial femoral arteries [25]. In addition, the ovine model has a coagulation and fibrinolytic system with more similarities to that of humans than other species [25]. In this model, the majority of the base braid in the 75:25/PLCL device was absorbed by 6 months, similar to the findings from the swine model. As expected, the 85:15/PLCL base braid demonstrated a longer resorption time compared to the $75: 25 / \mathrm{PLCL}$, with full resorption by 18 months. In both designs, mild to moderate amount of inflammation without granuloma was present, minimal vessel injury was observed, and the EEL was fully intact at all time points, indicating again acceptable vascular compatibility from implantation through resorption (Figure 6B and Supplemental Table 3). The 85:15/PLCL optimized implant design is biocompatible and addresses elastic recoil of the blood vessel by maintaining mechanical properties for 3 months.

In summary, we have developed a unique composite design to create a bioresorbable, self-expanding implant consisting of an elastomer coating that can be applied to a base braid to provide mechanical strength and to resist stress relaxation. Leveraging this composite design we have created an implant with acute properties similar to metal benchmark devices from "weak" polymer materials. The self-expansion and mechanical properties allow the implant to be delivered into and conform to various cavities within the body.

This composite device that is bioresorbable and self-expanding meets all the design requirements for vessels undergoing significant motion: it is strong, elastic, and biocompatible. Given that pre-clinical safety of the device has been established, the next step is to examine the device in human clinical studies. In particular, the chronic recoil performance of the implant in calcified lesions could be a limitation and needs to be evaluated. This device has the potential to be the first bioresorbable, fully self-expanding implant, affording patients an alternative treatment option.

The applicability of the composite strategy described herein goes beyond the specific bioresorbable device described herein. Bioresorbable implants eliminate any permanent nidi for chronic irritation. The coupling of strong materials with elastomeric ones provides an approach to create bioresorbable constructs that mimic the properties of native soft tissue. This approach has tremendous potential in the development of new implants to treat diseases of soft tissue across the body. 
References:

329 1. Nishio, S., et al. Long-Term (>10 Years) clinical outcomes of first-in-human biodegradable poly-I-lactic acid coronary stents: Igaki-Tamai stents. Circulation. 125, 2343-53 (2012).

2. Bunger, C.M., et al. Sirolimus-eluting biodegradable poly-L-lactide stent for peripheral vascular application: a preliminary study in porcine carotid arteries. J. Surg. Res. 139, 77-82 (2007).

3. Uurto, I., et al. Drug-eluting biodegradable poly-D/L-lactic acid vascular stents: an experimental pilot study. J. Endovasc. Ther. 12, 371-9 (2005).

4. Onuma, Y., et al. Three-year results of clinical follow-up after a bioresorbable everolimus-eluting scaffold in patients with de novo coronary artery disease: the ABSORB trial. Eurolntervention. 6, 447-53 (2010).

5. Rapoza, R. BVS Technology: Understanding the Principle of Bioabsorbable DES, in Innovations in Cardiovascular Interventions. Tel Aviv, Israel (2008).

6. Scheinert, D., et al. Prevalence and clinical impact of stent fractures after femoropopliteal stenting. J. Am. Coll. Cardiol. 45, 312-5 (2005).

7. van der Giessen, W.J., et al. Marked inflammatory sequelae to implantation of biodegradable and nonbiodegradable polymers in porcine coronary arteries. Circulation. 94, 1690-7 (1996).

8. Lincoff, A.M., et al. Sustained local delivery of dexamethasone by a novel intravascular eluting stent to prevent restenosis in the porcine coronary injury model. J. Am. Coll. Cardiol. 29, 808-16 (1997).

9. Fischell, T.A. Polymer coatings for stents. Can we judge a stent by its cover? Circulation. 94, 1494-5 (1996).

10. Venkatraman, S., Boey, F., Lao, L.L. Implanted cardiovascular polymers: Natural, synthetic and bio-inspired Prog. Polym. Sci. 33, 853-874 (2008).

11. Vogt, F., et al. Long-term assessment of a novel biodegradable paclitaxel-eluting coronary polylactide stent. Eur. Heart J. 25, 1330-40 (2004).

12. Chemical \& Physical Properties of Select Polymers [cited May 26, 2014]; http://www.absorbables.com/technical/properties.html

13. Nuutinen, J.P., et al. Mechanical properties and in vitro degradation of bioresorbable knitted stents. J. Biomater. Sci. Polym. Ed. 13, 1313-23 (2002).

14. Chen, M.C., et al. Rapidly self-expandable polymeric stents with a shape-memory property. Biomacromolecules. 8, 2774-80 (2007).

15. Nuutinen, J.P., et al. Mechanical properties and in vitro degradation of bioabsorbable self-expanding braided stents. J. Biomater. Sci. Polym. Ed. 14, 255-66 (2003).

16. Pulapura, S. and J. Kohn. Trends in the development of bioresorbable polymers for medical applications. J. Biomater. Appl. 6, 216-50 (1992).

17. Chen, C.C., et al. Preparation and characterization of biodegradable PLA polymeric blends. Biomaterials. 24, 1167-73 (2003).

18. Vert, M., et al. Bioresorbability and biocompatibility of aliphatic polyesters. J. Mater. Sci. Mater. Med. 3, $432-446$ (1992).

19. Orchel, A., et al. Growth of human chondrocytes on biodegradable synthetic polymers. Acta Pol. Pharm. 63, 455-6 (2006).

20. Wise, D.L., Biomaterials and Bioengineering Handbook (CRC Press, Boca Raton 2001).

21. Juan, V.C.-R., et al., Advances in Biomaterials Science and Biomedical Applications Ch. 3: Degradation of Polyurethanes for Cardiovascular Applications. (InTech, Rijeka, Croatia, 2013).

22. Dalene, M., G. Skarping, and T. Brorson. Chromatographic determination of amines in biological fluids with special reference to the biological monitoring of isocyanates and 
amines. IV. Determination of 1,6-hexamethylenediamine in human urine using capillary gas chromatography and selective ion monitoring. J. Chromatogr. 516, 405-13 (1990).

23. Dalene, M., G. Skarping, and H. Tinnerberg. Biological monitoring of hexamethylene diisocyanate by determination of 1,6-hexamethylene diamine as the trifluoroethyl chloroformate derivative using capillary gas chromatography with thermoionic and selective-ion monitoring. J. Chromatogr. B. Biomed. Appl. 656, 319-28 (1994).

24. Serruys, P.W., et al. Incidence of restenosis after successful coronary angioplasty: a time-related phenomenon. A quantitative angiographic study in 342 consecutive patients at 1, 2, 3, and 4 months. Circulation. 77, 361-71 (1988).

25. Leigh Perkins, L.E. Preclinical Models of Restenosis and Their Application in the Evaluation of Drug-Eluting Stent Systems. Vet. Pathol. 47, 58-76 (2010).

402

\section{Acknowledgements:}

We thank Drs. Jim Anderson, Renu Virmani, Rob Schwartz, and Steven Hilbert for consultation and feedback. We acknowledge the efforts of Dr. Alex Pappas, Dr. Tatfong Ng, Dr. Kicherl Ho, Dr. Irina Gitlin, Dr. Parisa Zamiri, Dr. Wendy Naimark, Adam Rago, Dhyan Sundaresh, John Marini, Sara Morneau, My Le, Saramma Varughese, and Kimberly Un in device fabrication, data collection, analytical evaluation, and manuscript review.

\section{Author Contributions:}

U.S., D.C., L.C., Y.K., C.Y., G.Z., R.B., S.W., and M.P. conceived the experiments. C.Y. and J.M. performed the experiments. U.S., D.C., L.C., Y.K., C.Y., Q.P., G.Z. R.B. S.W. undertook analysis of the data and results. U.S., D.C., L.C., Y.K., C.Y, Q.P., R.L., and G.W. wrote the manuscript

404 All authors, including Drs. Whitesides and Langer, have stock options in 480 Biomedical 
407 To synthesize the elastomer pre-polymer, a catalyst Sn (Oct) $)_{2}$, initiator pentaerythritol, and the 408 monomers were added to a round-bottom flask at the desired ratio (e.g., for the poly (glycolideco-caprolactone) (PGCL) prepolymer, the flask was charged successively with $\mathrm{Sn}(\mathrm{Oct})_{2}(10.5 \mathrm{mg})$, pentaerythritol (300mg), glycolide $(30.0 \mathrm{~g})$, and $\varepsilon$-caprolactone $(30.0 \mathrm{~g})$ ). The reaction proceeded at $170^{\circ} \mathrm{C}$ under a nitrogen atmosphere for $24 \mathrm{~h}$. The resulting pre-polymer was precipitated, washed thoroughly and dried. $\mathrm{A}{ }^{1} \mathrm{H} \mathrm{NMR}$ in $\mathrm{CDCl}_{3}$ was acquired to measure the lactide:caprolactone mol:mol polymer composition, and a gel permeation chromatograph (GPC) using poly(methyl methacrylate) as standards was acquired to measure the $M_{n}, M_{w}$, and the polydispersity index (PDI).

416 Films of the PGCL elastomer were prepared by dissolving the PGCL pre-polymer (1.0g) with hexamethylene diisocyanate at various concentrations in $20 \mathrm{~mL}$ of dichloromethane (DCM). The solution was poured into a $10 \mathrm{~cm}$ aluminum pan, dried, and cured at $100^{\circ} \mathrm{C}$ for 16 hours. The elastomer films were cut into dog bone-shaped coupons $3.18 \mathrm{~mm}$ in width for Instron testing. The coupons were loaded onto the grips with a separation of $15 \mathrm{~mm}$ and the stress-strain responses of the films were measured at room temperature.

\section{Fabrication of the devices}

423 Fabrication of the implants required several steps: (1) braiding of the base braid, (2) spray coating the elastomer pre-polymer onto the base braid, and (3) curing the elastomer. To fabricate the base braid, poly(L-lactide-co-glycolide) (L-PLGA) resins purchased from Corbion (Gorinchem, The Netherlands) were first melt-extruded into fibers at Biogeneral, Inc (San Diego, CA, USA). These fibers were spooled onto 32 individual bobbins and then braided along a mandrel. Braids were annealed on the mandrels under tension ( 30 minutes at 95 or $120^{\circ} \mathrm{C}$ for $75: 25$ and 10:90, respectively and 22 hours at $130^{\circ} \mathrm{C}$ for $85: 15$ ) and then stored frozen until use. At the time of use, braids were removed from the mandrel, cut to the desired length, and radiopaque marker bands (platinum/tungsten) were manually placed on the ends of the device.

432 For spray coating, the braid was mounted onto a specially designed holding fixture that enabled complete coating of the braid fibers. The elastomer pre-polymer and crosslinker (hexamethylene diisocyanate, $\mathrm{HDI}$ ) were dissolved in dichloromethane (e.g., $10 \mathrm{~g}$ of PGCL prepolymer and 1.87 $\mathrm{mL}$ of $\mathrm{HDI}$ in $200 \mathrm{~mL}$ of solvent). This solution was spray coated to achieve a conformal coating of the braid. The braid was rotated and horizontally translated throughout the spraying process to ensure a uniform coating along the length of the device. The mass of the elastomer was optimized to achieve the desired mechanical properties (Supplemental Figure 5). After spray coating of the elastomer, the holding fixture with the coated braid was placed in an oven and heated at elevated temperatures $\left(100^{\circ} \mathrm{C}\right.$ were used for the $10: 90,75^{\circ} \mathrm{C}$ for the $75: 25$ and a staged heating system of $75^{\circ} \mathrm{C}$ and then $100^{\circ} \mathrm{C}$ for $\left.85: 15\right)$. 
443 In order to characterize the self-expanding, bioresorbable device, we measured the acute radial stiffness and the diameter after simulated deployment. The RRF and COF were quantified using a Radial Force Gauge (Machine Solutions, Flagstaff, AZ). Test articles were placed into a cylindrical 'iris' fixture which compressed the implant to a 7French diameter, then increased in diameter to allow the implant to expand. Measurements were taken at nominal implant diameter minus $1.5 \mathrm{~mm}$ (i.e. target vessel diameter) during the compressive and expansive parts of the cycle; RRF as the diameter decreased, and COF as the diameter increased. Testing on benchmark devices was performed on an $n=2$ or 3 , while testing on elastomer coated braids was performed on a minimum of an $n=3$ samples. Dimensional scaling of the radial forces of our implant at $6 \mathrm{~mm}$ nominal diameter to the $7 \mathrm{~mm}$ nominal diameter of the benchmark devices was performed for. A Student's t-test was used to determine significant differences in radial stiffness between the elastomer-coated implant and each of the other test articles. Samples for characterization of retention of mechanical properties $(n=5)$ were placed in phosphate-buffered saline (PBS) and at designated timepoints, were removed, dried, and subsequently tested as described above.

Implant diameter was measured after simulated deployment using a laser micrometer as a measurement of recovery. Test samples were loaded into 7French delivery catheters, flushed with (PBS) for $10 \mathrm{~min}$, then placed in a $37^{\circ} \mathrm{C}$ PBS bath for $10 \mathrm{~min}$, simulating delivery in the body. After simulated loading and delivery, test samples were deployed into a $37^{\circ} \mathrm{C}$ bath, removed from the bath, and dried. Within one minute of deployment, implant outer diameter was measured using a laser micrometer in six locations.

Fatigue testing was performed on $85: 15 /$ PLCL designs to evaluate cyclic loading on implant integrity. Implants were deployed into $5.5 \mathrm{~mm}$ diameter compliant silicone tubing and then subjected to cyclic bending by a 1.5 inch radius drum at a frequency of 1 hertz. Visual observations were made on a weekly basis for signs of fracture or failure. Additionally, the invessel lengths of the implants were measured as a surrogate for implant failure. The relevance of this measurement is that, as a consequence of recoil, the implant diameter will decrease resulting in an increase in its length (i.e. the implant forelengthens as it becomes smaller in diameter). By monitoring length, sudden reductions can be detected, suggesting the implant mechanically fails in such a way that the vessel elastically springs back to its original length. Description of accelerated resorption assay

Implants were placed unconstrained individually in glass vials filled with $20 \mathrm{~mL}$ of phosphate/citrate/borate buffer solution at a $\mathrm{pH}=12.0$. The vials were placed in a shaking water bath at $37^{\circ} \mathrm{C}$. At a designated time point, the implants were removed from the solution, rinsed with de-ionized water, and dried under vacuum until a constant weight was obtained. The \% mass loss at that time point is calculated as outlined in equation below. The mass loss experiments were terminated once the implants lost their integrity.

$$
\% \text { Mass Loss }=\frac{\text { Initial Scaffold Mass }- \text { Scaffold Mass at Designated Timepoint }}{\text { Initial Implant Mass }} \times 100
$$


Data demonstrated that one day under accelerated condition $\left(\mathrm{pH} 12,37^{\circ} \mathrm{C}\right)$ roughly was equal to 8.5 days under physiological conditions $\left(\mathrm{pH} 7.4,37^{\circ} \mathrm{C}\right.$ ) (Supplemental Figure 6 ).

\section{Pre-clinical assessment}

484 Two preclinical studies were conducted to assess the biocompatibility of various implant designs. 485 The first study evaluated 10:90/PGCL, 75:25/PGCL, and 75:25/PLCL designs in healthy nonatherosclerotic Yucatan mini/hybrid farm swine while the second study evaluated 75:25/PLCL and 85:15/PLCL designs in adult Suffolk Cross-bred sheep. Full characterization data of these implant designs can be found in Supplemental Table 4. All animal work was performed under the oversight by the Comité institutionnel de protection des animaux d'AccelLAB and was insured compliance with the Canadian Council on Animal Care. Devices were fabricated to a nominal diameter of 6 or $7 \mathrm{~mm}$ and a length of $20 \mathrm{~mm}$. Immediately prior to implantation, the implants were loaded into a 9.5French catheter-based delivery system and were delivered to the target vessels. Vessel sizes were selected to ensure sufficient vessel bump-out (i.e., to ensure the device did not interfere blood flow and to control vessel injury due to chronic outward force). Implant-to-artery ratio was targeted to between 1.15 and 1.25 for the swine study and between 1.00 and 1.15 for the sheep study. Animals were euthanized at desired time-points between 1-18 months. There was at least an $n=4$ samples for each group tested at each timepoint. After sacrifice, implants were harvested and preserved in formalin, dehydrated, and embedded in paraffin. Three crosssections (proximal, middle, and distal) for each implant were cut using a microtome, and stained for histopathological evaluation. The semi-quantitative scoring scheme for injury, inflammation, and fibrin are described in Supplemental Table 5. The preclinical proof-of-concept studies were conducted to efficiently utilize the number of available animals with the goal of providing directional information for iterating on implant design. Therefore, the studies were not

505 Data Availability Statement

506 The authors declare that all relevant data supporting the finding of this study are available within 507 the paper and its Supplementary Information files. Additional data are available from the 508 corresponding author upon request. 
A.

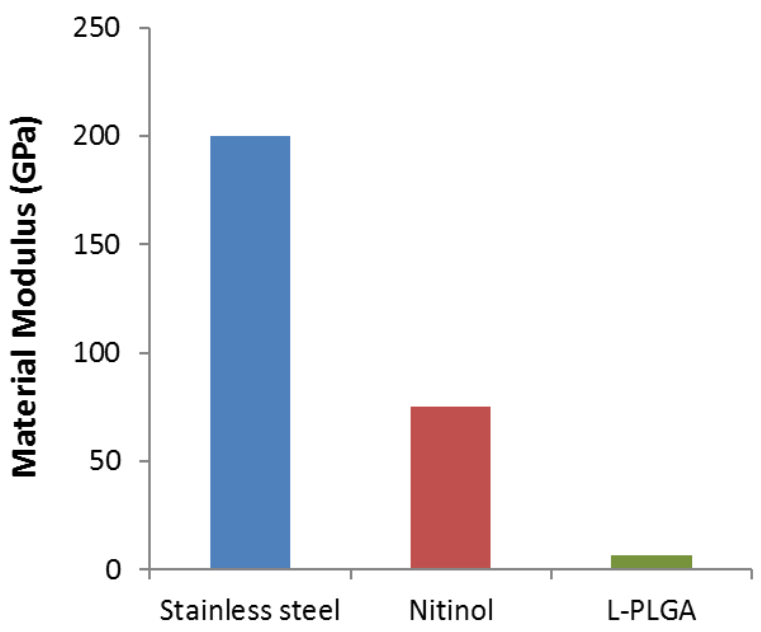

B.

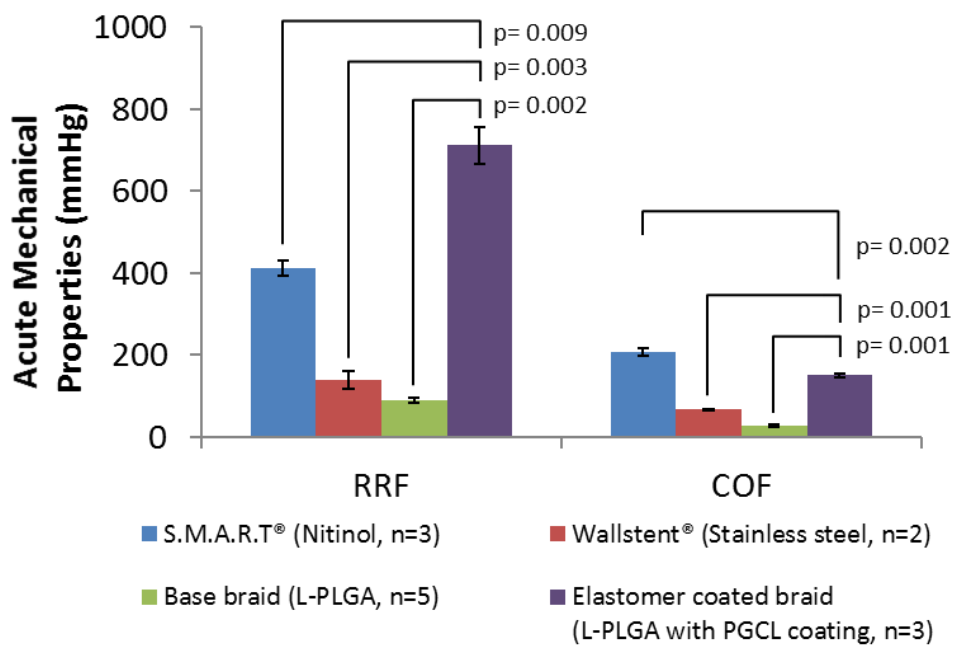

510 Figure 1. Mechanical properties of the bioresorbable, self-expanding implant. (A) Comparison of 511 the modulus of materials used in development of self-expanding stents. (B) Comparison of 512 mechanical properties of the Wallstent ${ }^{\circledR}$ (stainless steel), S.M.A.R.T.® (Nitinol), and elastomer 513 coated braid (L-PLGA coated with PGCL). A two-sided Student's t-test was used to determine 514 significant differences in radial stiffness between the elastomer-coated implant and each of the 515 other test articles. 

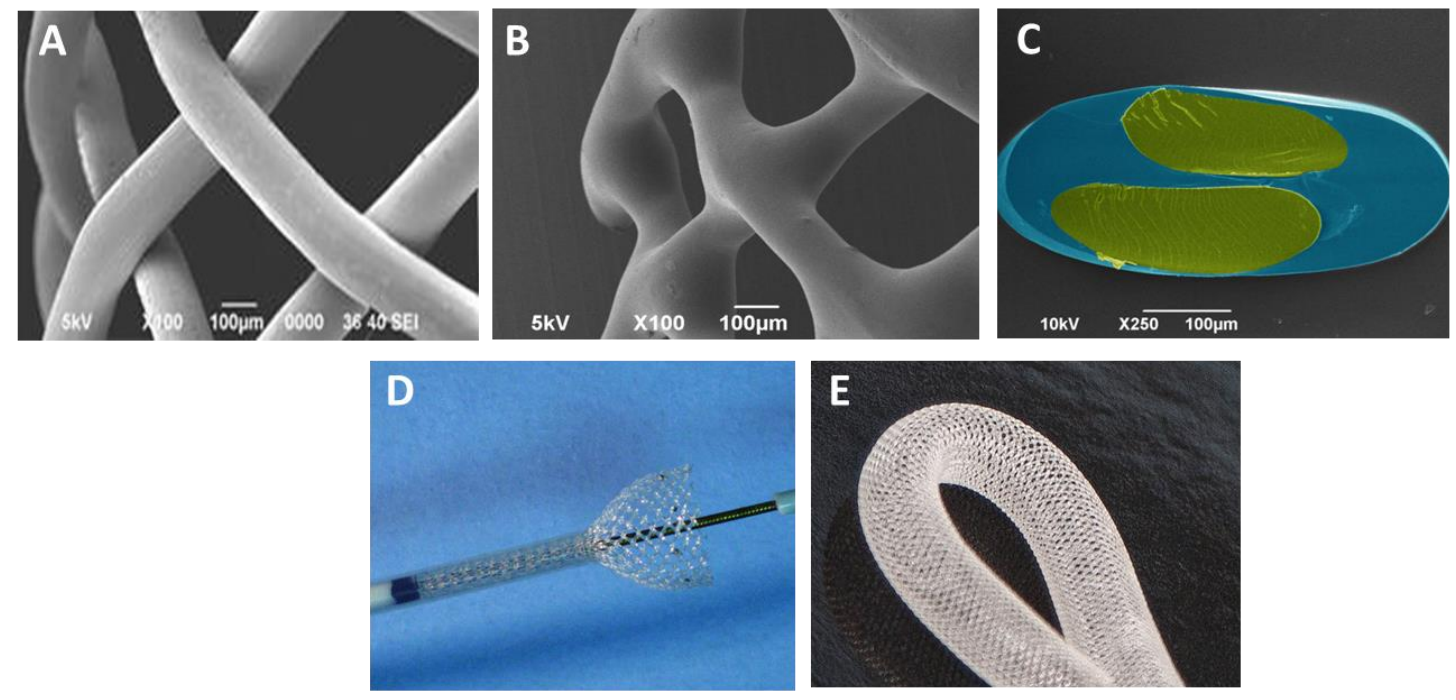

$\mathbf{F}$
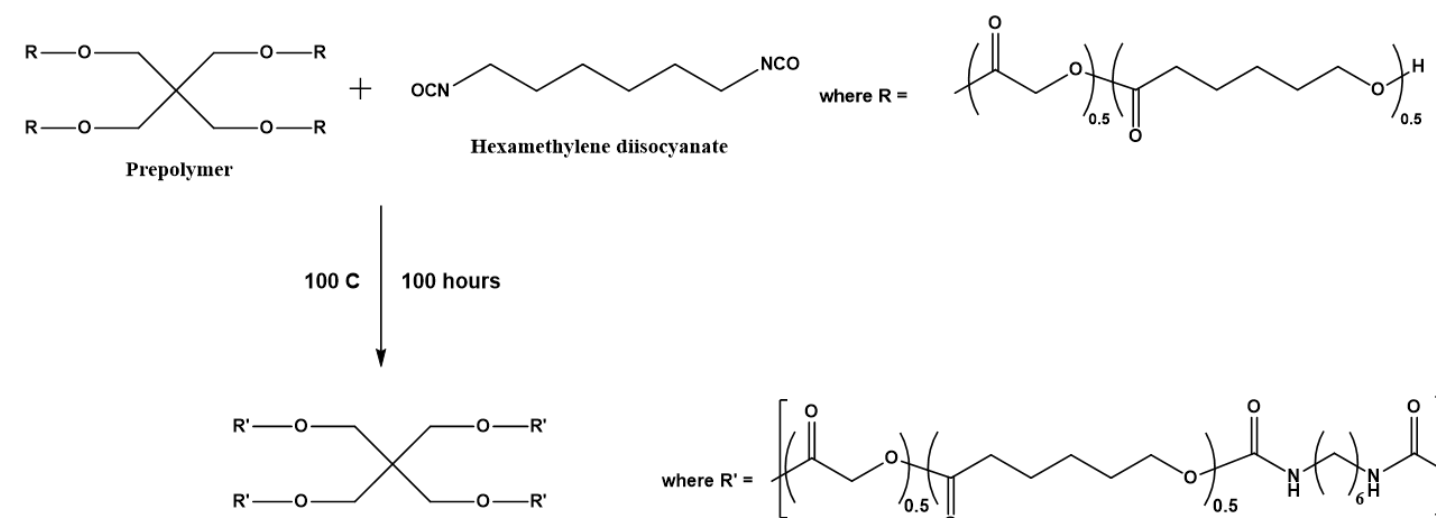<smiles>CCC(=O)C(C)(C)NC(=O)C(C)(C)OCCCCCC(=O)C(C)(C)OCC(=O)C(C)(C)CC</smiles>

Thermoset polyester elastomer with urethane crosslinks

517 Figure 2. Creation of the strong, elastic, resorbable, self-expanding implant. (A) Scanning

518 electron micrograph (SEM) of a bioresorbable PLGA base braid. (B) SEM of base braid after the 519 elastomer coating has been applied. This elastomer provides a mechanism for the fibers to 520 return to nominal diameter, imparting strength on the device. (C) False-colored SEM of a cross521 section of the intersection point of the elastomer-coated braid. The yellow areas represent the 522 base braid and the blue area is the elastomer coating. (D) Photograph illustrating self-expansion 523 of implant as it is deployed from a 9Fr catheter. (E) Photograph of the implant demonstrating 524 flexibility of the design. (F) Chemical reaction of the four-arm PGCL prepolymer and HDI to 525 create the bioresorbable elastomer. 
A.
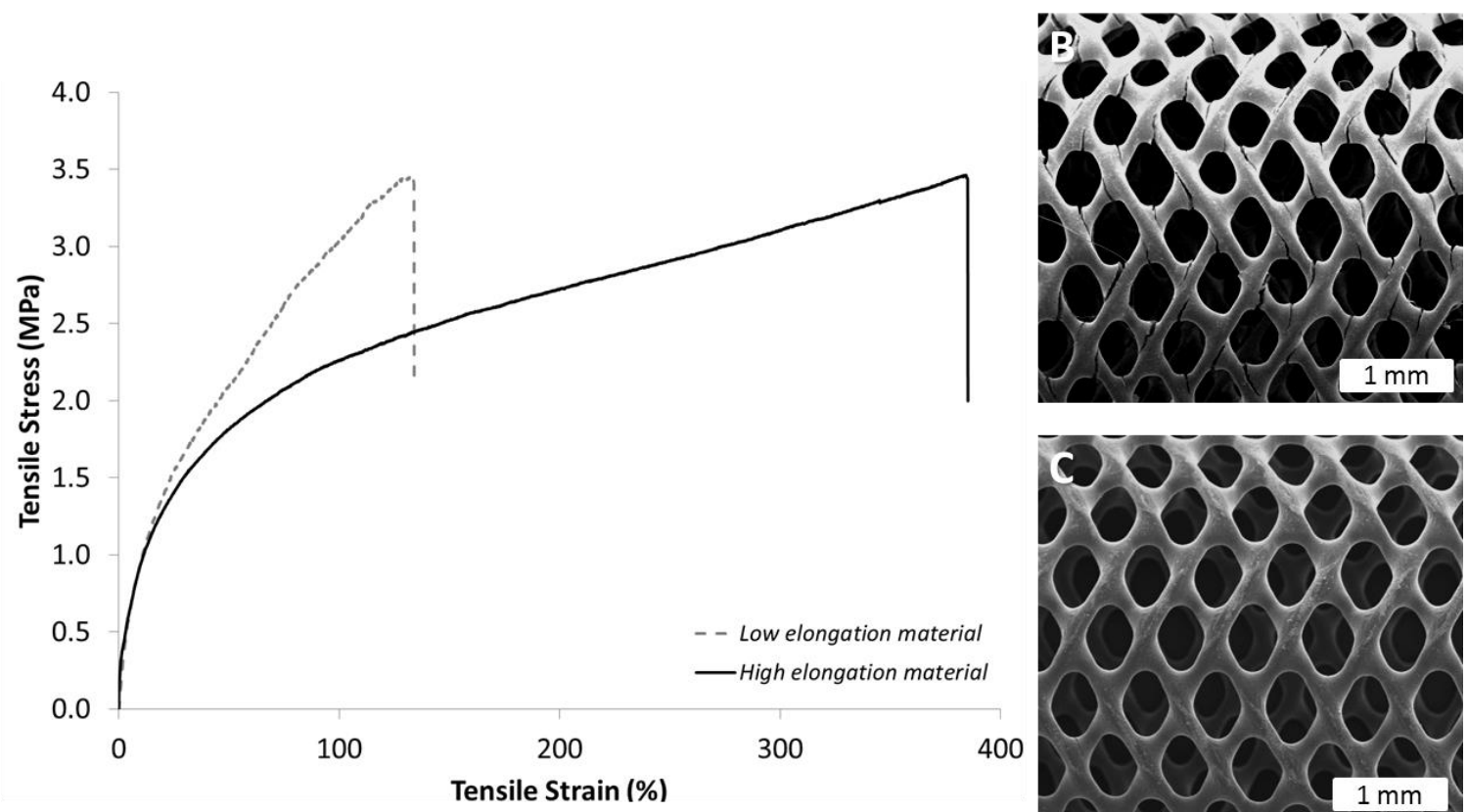

527 Figure 3. Mechanical properties and morphology of low and high elongation material. (A) 528 Representative stress - strain curves of films for two PGCL elastomers: one with high and one with low elongation-to-break. (B) Scanning electron micrograph depicting cracked morphology of a low elongation-to-break elastomer applied to a base braid and subsequently crimped and deployed. (C) Scanning electron micrograph depicting non-cracked morphology of a high 
1 mo
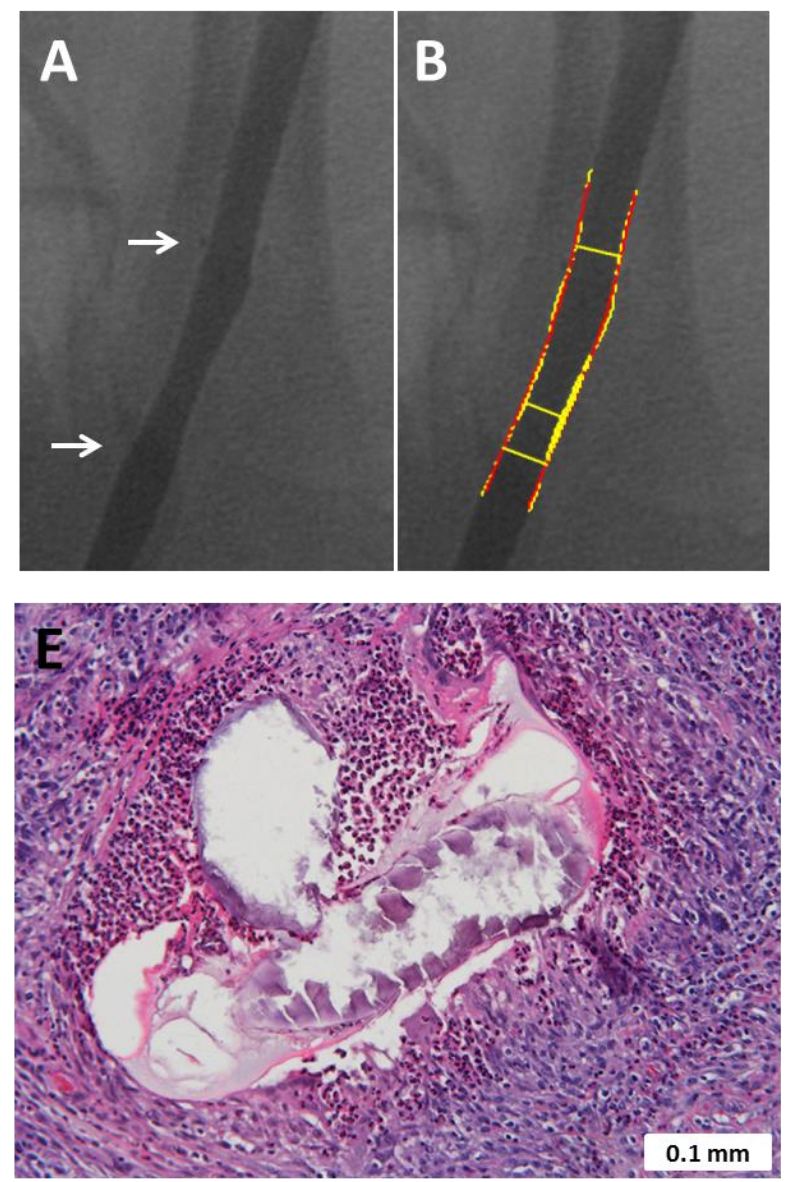

3 mo
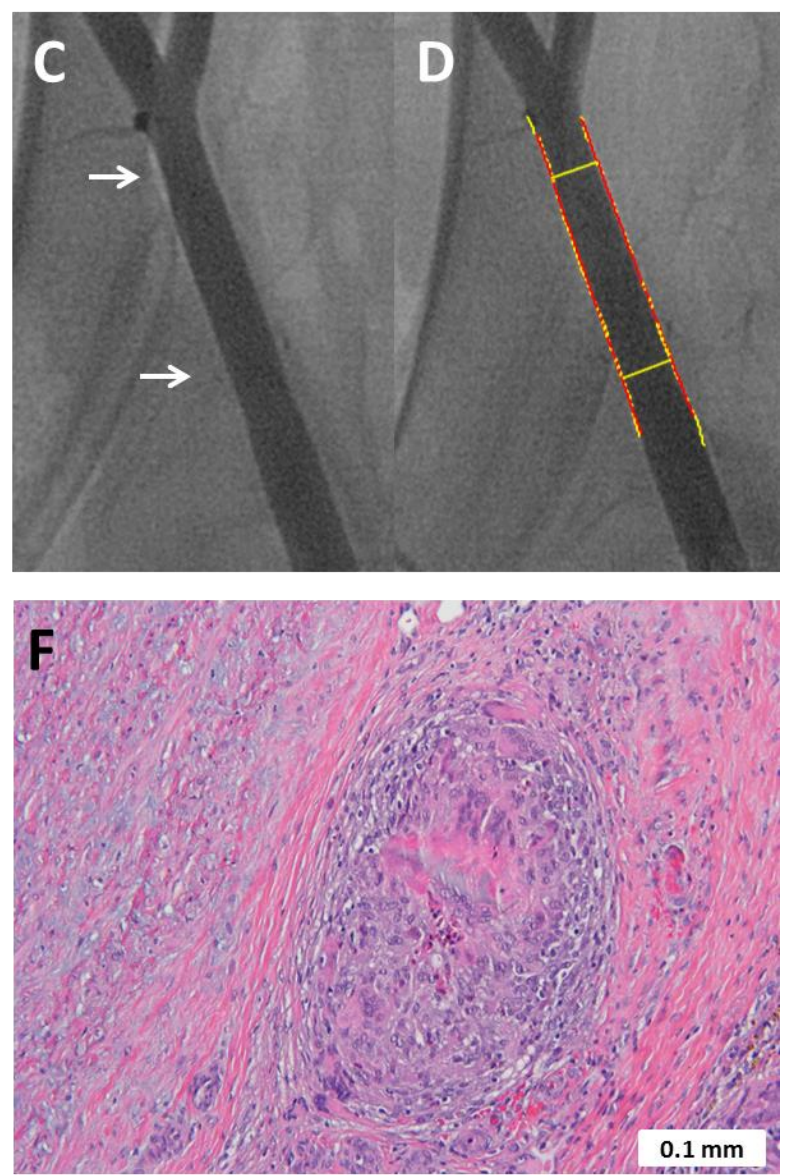

534 Figure 4. Testing of 10:90/PGCL implant in vivo in swine ilio-femoral vessels. (A - D) 535 Angiography images depicting vessel patency at 30 and 90 days. (E - F) Histology images 536 stained with hematoxylin and eosin demonstrating a severe inflammatory response at 30 days, 537 with some resolution by 90 days. 


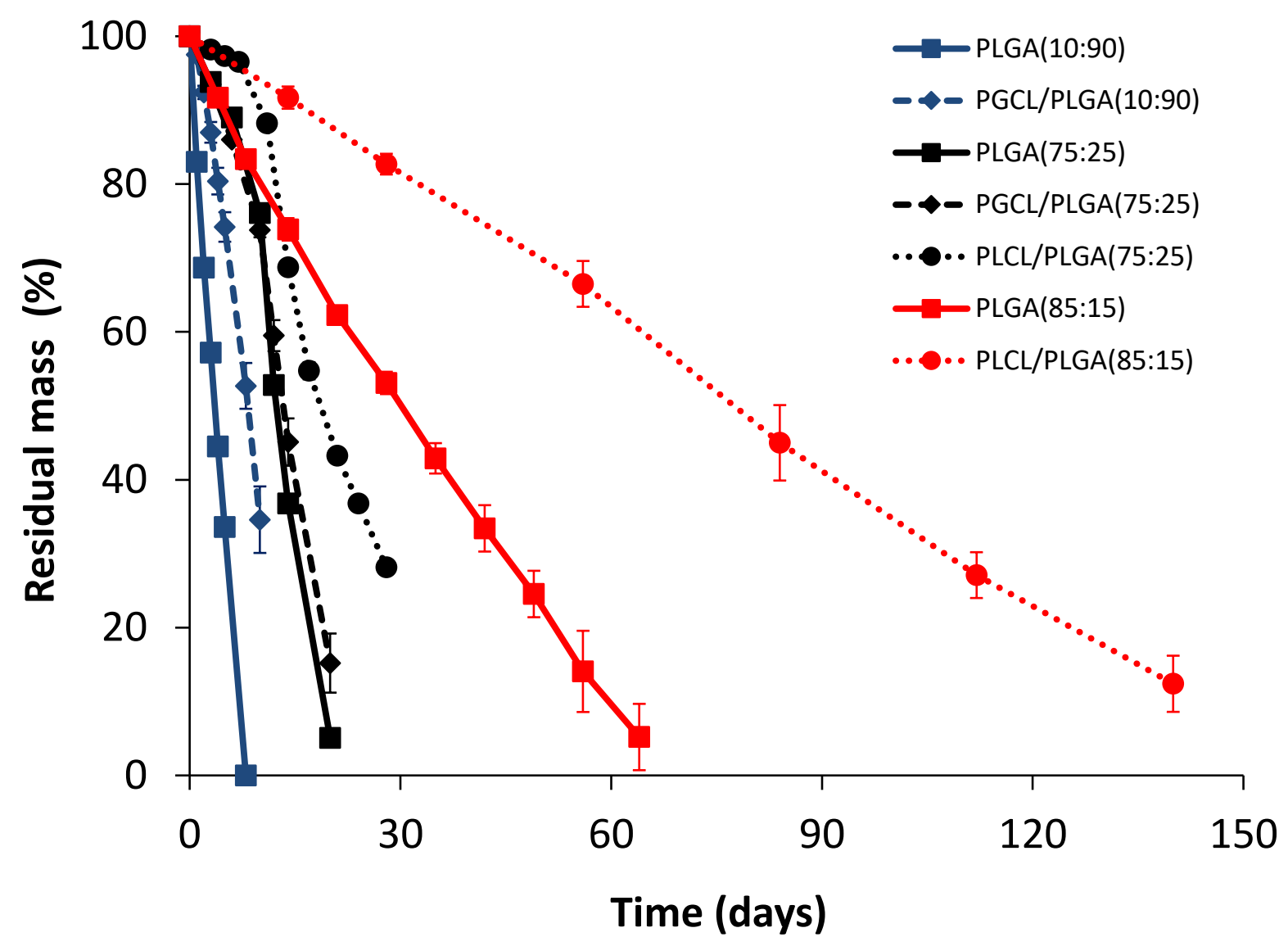

539 Figure 5. Accelerated in vitro resorption time of base braids and elastomer-coated implants.

540 Comparison of residual mass of 10:90 L-PLGA, 75:25 L-PLGA, and 85:15 L-PLGA base braids

541 with their corresponding elastomer coated devices (10:90/PGCL, 75:25/PGCL, 75:25/PLCL, and 542 85:15/PLCL). Each day represents approximately 1 week in real time resorption. Each data 543 point represents the mean and standard deviation on an $n=3$ to 5 samples. 
A
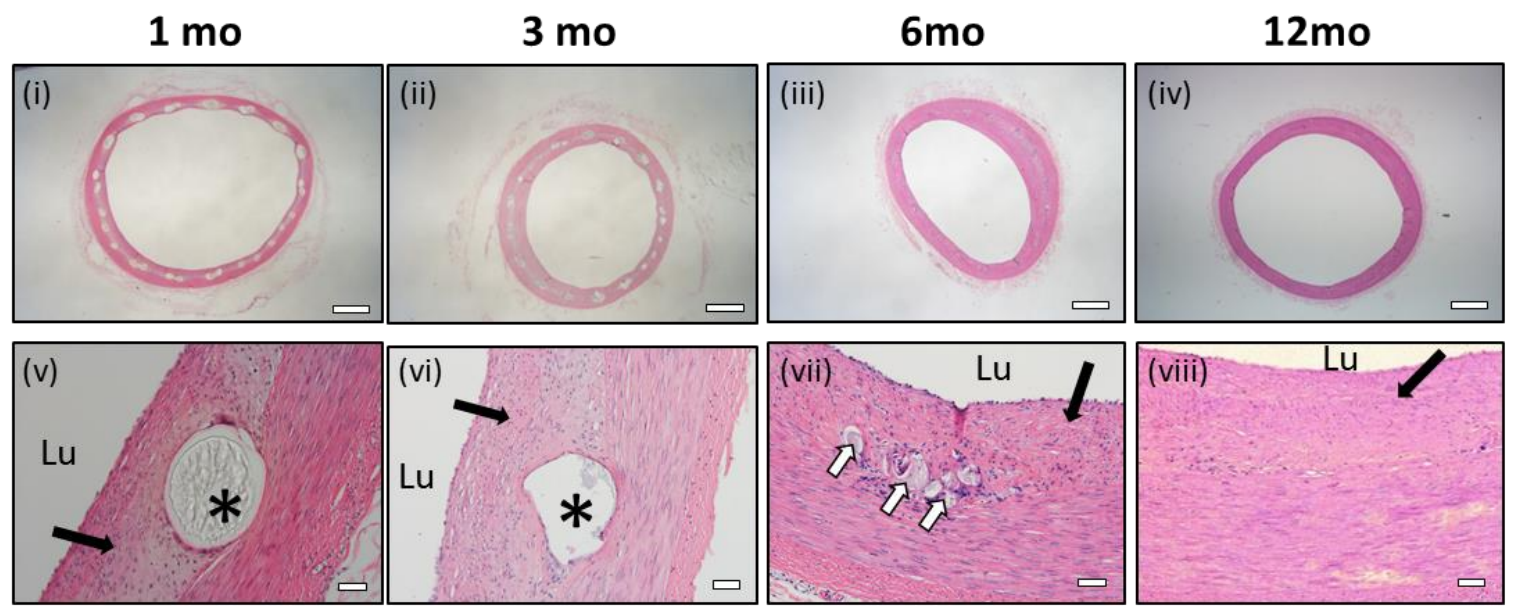

B

3 mo
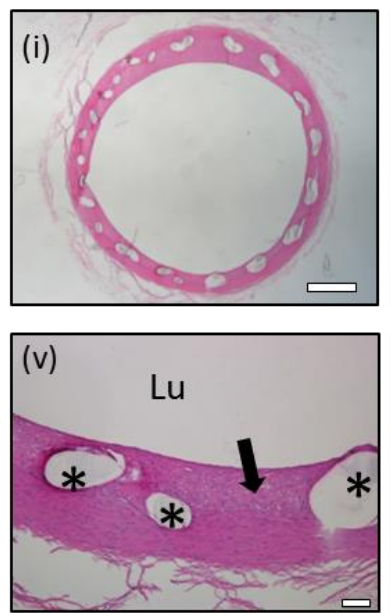

$6 \mathrm{mo}$
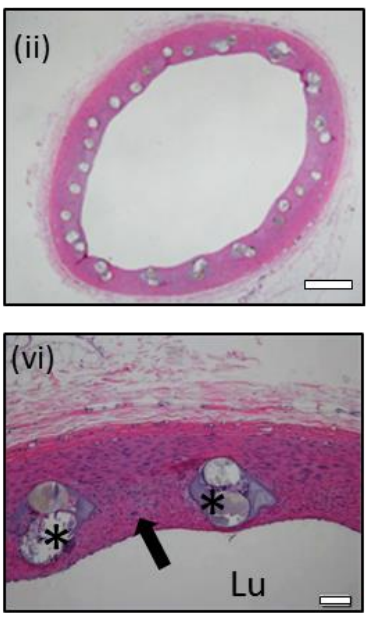

$12 \mathrm{mo}$
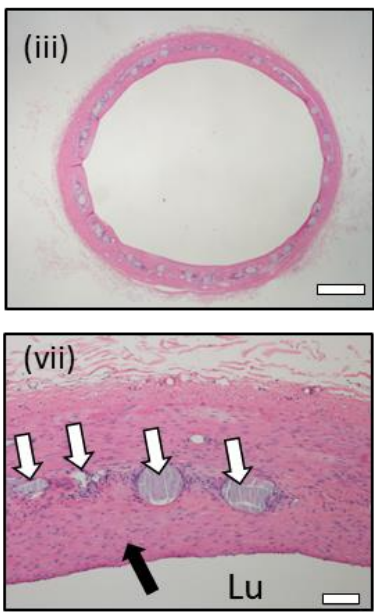

$18 \mathrm{mo}$
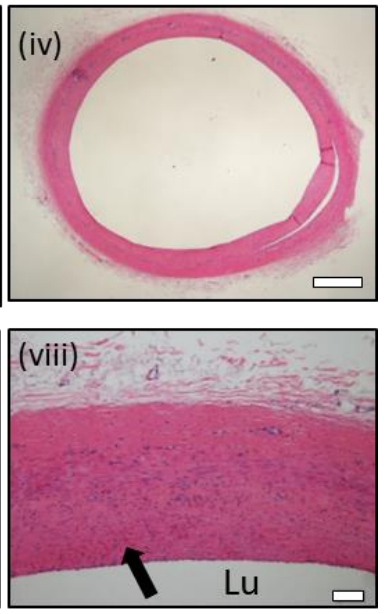

Figure 6. Histological results of elastomer-coated implants through full resorption. (A) Hematoxylin and eosin stained images of 75:25/PLCL implants in vivo in swine ilio-femoral vessels. (i-iv) Low magnification histology images depicting vessel patency and time course of resorption. Scale bar represents $1 \mathrm{~mm}$. (v-viii) High magnification histology images showing time course of inflammatory response and resorption of individual struts. Scale bar represents $50 \mu \mathrm{m}$. (B) Hematoxylin and eosin stained images of 85:15/PLCL implants in sheep superficial

551 femoral or profunda arteries. (i-iv) Low magnification images depicting vessel patency and resorption over time. Scale bar represents $1 \mathrm{~mm}$. (v-viii) High magnification images showing time course of inflammatory response and resorption of individual struts. Scale bar represents

$554100 \mu \mathrm{m}$. For all images, the asterisks indicate implant struts; white arrows show remnants of 555 polymer material; black arrows point to neointimal tissue; Lu indicates the lumen. 